\title{
Establishing Spatial Correspondence between the Inner Colon Surfaces from Prone and Supine CT Colonography
}

\author{
Holger Roth ${ }^{1}$, Jamie McClelland ${ }^{1}$, Marc Modat ${ }^{1}$, Darren Boone ${ }^{2}$, \\ Mingxing $\mathrm{Hu}^{1}$, Sebastien Ourselin ${ }^{1}$, Greg Slabaugh ${ }^{3}$, \\ Steve Halligan ${ }^{2}$, and David Hawkes ${ }^{1}$ \\ ${ }^{1}$ Centre for Medical Image Computing, University College London, UK \\ ${ }^{2}$ Department of Specialist Radiology, University College Hospital, London, UK \\ ${ }^{3}$ Medicsight PLC, London, UK
}

\begin{abstract}
Colonography is an important screening tool for colorectal lesions. This paper presents a method for establishing spatial correspondence between prone and supine inner colon surfaces reconstructed from CT colonography. The method is able to account for the large deformations and torsions of the colon occurring through movement between the two positions. Therefore, we parameterised the two surfaces in order to provide a $2 \mathrm{D}$ indexing system over the full length of the colon using the Ricci flow method. This provides the input to a non-rigid B-spline registration in $2 \mathrm{D}$ space which establishes a correspondence for each surface point of the colon in prone and supine position. The method was validated on twelve clinical cases and demonstrated promising registration results over the majority of the colon surface.
\end{abstract}

\section{Introduction}

Colorectal cancer is one of the main types of cancer, leading to more than 630,000 deaths worldwide each year [1. Traditional colonoscopy using a video endoscope can produce miss rates of up to $27 \%$ for adenomas smaller or equal to $5 \mathrm{~mm}[2]$. Furthermore, it can cause significant discomfort and is not without risk of perforating the colon. These drawbacks have led to the development of alternative screening methods such as computed tomography colonography, which is becoming established as a standard screening tool for the detection and diagnosis of colorectal lesions in the USA, Europe and Japan. The bowel is cleansed before the procedure by administering a powerful laxative and inflated. CT images are taken in both prone and supine positions, resulting in a large deformation of the colon. Remaining faecal material and fluids can be tagged with contrast agent and removed digitally. However, faecal remnants or folds of the colonic wall can still mimic the appearances of polyps, leading to false positives in the diagnosis. Routinely, the radiologist establishes the spatial correspondence between the two views by eye which is a difficult task for even the most experienced radiologist and can thus introduce delays and errors. An automatic method of establishing correspondence between the colon surfaces visualised in prone and supine

T. Jiang et al. (Eds.): MICCAI 2010, Part III, LNCS 6363, pp. 497-504, 2010.

(C) Springer-Verlag Berlin Heidelberg 2010 
CT images would have the potential to save time and improve accuracy during the diagnostic process. Furthermore, it could improve computer aided detection (CAD) algorithms.

Registration of prone and supine CT images of the colon has been attempted by aligning extracted centrelines of both views [3] as an index of location. However, this method essentially provides only one degree of freedom of location within the colonic surface and does not account for torsion. Another approach is to define several anatomical landmarks [4, like anus, cecum and flexures, in order to align both colons. However, flexures are difficult to locate accurately and the identification of only a small number of points is insufficient to describe the complex folding and deformation of the colon between prone and supine positions. A voxel-based method was proposed by Suh et al. [5], which also uses the centrelines to generate an initial deformation field and then treats the registration task as an optical flow process. However, it is clear from our own studies that the changes in shape and location of anatomy between prone and supine CT colonography mean that conventional image intensity-based non-rigid registration algorithms are not sufficiently robust or accurate. Lamy et al. [6] use the teniae coli as an additional feature producing a deformation field with a rotational component. However, teniae coli are difficult to extract over the full length of the colon and cannot describe the deformation of the whole colonic surface.

We propose a new method based on a $2 \mathrm{D}$ manifold representing the internal colon lumen surface which is able to account for the large deformations and torsion of the colon in both positions. The colon is a tube and the internal surface can be mapped onto a cylinder with two indices describing any surface location. Each location corresponds to a $3 \mathrm{D}$ point in the CT scan and can act as an index to a rich set of both surface and volume features. Our method uses these features from the $3 \mathrm{D}$ data to drive the registration, but finds a transformation between the $2 \mathrm{D}$ manifolds representing the colon surface in the prone and supine scans. Hence, this greatly eases the registration in comparison to full 3D registrations.

To the best of our knowledge nobody else has proposed performing non-rigid registration on the $2 \mathrm{D}$ manifold in order to establish a full correspondence between all points on the $3 \mathrm{D}$ colonic surface.

\section{Methods}

A number of groups have proposed methods for unfolding the colon in order to produce $2 \mathrm{D}$ images of the inside of the colon. These images are usually produced in order to enable better examination of the colon surface and to aid detection of polyps [7. Conformal mappings can provide a one-to-one mapping between the $3 \mathrm{D}$ surface and the $2 \mathrm{D}$ image while minimising the necessary deformation [8]. A Surface $S_{1}$ in $R^{3}$ can be represented using a one-to-one mapping $\phi_{1}$ to $D_{1}$ in $R^{2}$. $S_{2}$ is mapped to $D_{2}$ through $\phi_{2}$ respectively. Hence, the transformation function $f$ between the three-dimensional surfaces $S_{1}$ and $S_{2}$ can be derived as shown in Fig. 11, where the principle is illustrated. $\tilde{f}$ is the registration $\tilde{f}: D_{1} \rightarrow D_{2}$ between the two flat surfaces. 


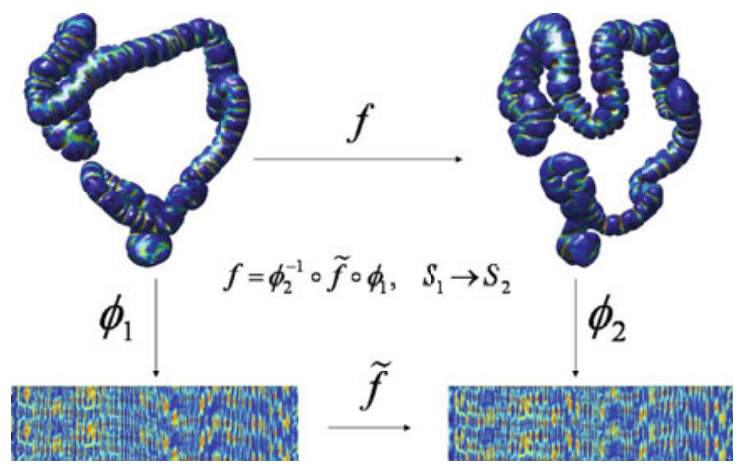

Fig. 1. The principle of surface registration using a 2D manifold, where the colour scale indicates the shape index (see section 2.3) at each coordinate of the surface computed from the $3 \mathrm{D}$ inner colonic surfaces $S_{i}$

\subsection{Colon Unfolding with Discrete Ricci Flow}

One recently developed technique for discrete surface parameterisation is based on Ricci. This method deforms a surface proportionally to its local curvature, where the curvature values evolve similarly to a heat diffusion process. It was first introduced by Hamilton [10, for Riemannian geometry. Qiu et al. 9] were the first to use this method to unfold the colon. The Ricci flow is defined as $\frac{d u_{i}(t)}{d t}=\bar{K}_{i}-K_{i}$, where $K_{i}$ is the Gaussian curvature at vertex $v_{i}, \bar{K}_{i}$ is the desired Gaussian curvature and $u_{i}$ is computed from a circle packing metric 8 . It can be shown that the Ricci flow is essentially the gradient flow of an energy function [11] which can be minimized using the gradient descent method. For the purpose of parameterisation of the colon surface, the target curvature should be zero for all vertices.

\subsection{Extraction of the Inner Colonic Surface}

We extracted triangulated meshes of the inner colonic surfaces $S_{i}$ using the segmentations of the air inside the prone and supine colons computed by the method described in [12. We ensured that the segmentations of the large intestine were topologically correct. In order to achieve that, some of the segmentations were manually edited. The segmentation is the input for a marching cubes algorithm with subsequent smoothing and decimation. This results in a closed and simply connected mesh along the air-to-tissue border in the CT-image.

To apply the Ricci flow method to the colon, each original genus-zero surface $S_{i}$ has to be converted to a genus-one surface $S D_{i}$ [8. Therefore, the inner colonic surface $S_{i}$ (which is topologically equal to a sphere) has to be converted to a torus-like surface. Hence, we remove faces of the surface mesh from cecum and rectum at user-identified positions. The remaining faces are duplicated with inverse orientation and joined at the boundaries of the previously produced holes. The resulting mesh $S D_{i}$ provides the input for the Ricci flow computation which provides the two-dimensional coordinates of each location within the surface. 


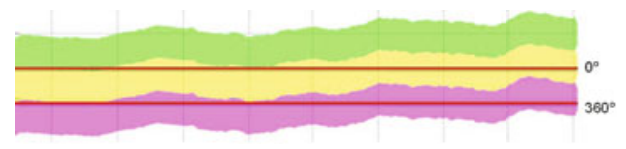

Fig. 2. Sampling the unfolded mesh (where the green and purple bands represent the repeat of the $2 \mathrm{D}$ coordinates of the surface) for a further 360 degrees clockwise and anticlockwise. The horizontal lines represent the re-sampled complete colon surface in a form suitable for registration

\subsection{Generating the 2D Coordinates of the Surface}

The Ricci flow algorithm converges to a planar surface with free border and local Gaussian curvature tending to zero everywhere by iteratively updating the edge lengths of the triangles. The optimisation carries on until the maximum difference between all $K_{i}$ and $\bar{K}_{i}$ is close enough to zero in order to produce a suitable parameterisation $D_{i}$ which can be embedded into planar $R^{2}$ space. This is computed in a similar manner to 8 where each planar triangle is computed based on its final edge length. As $D_{i}$ is not rectangular, we repeat the planar mesh so that a rectangular raster-image $I_{i}$ fully samples all points around the colon (as illustrated in Fig. 2). The top $\left(0^{\circ}\right)$ and bottom $\left(360^{\circ}\right)$ edges of the image correspond to the same point on the colon surface, thus representing the inner colonic surface as a cylinder. The horizontal axis $(x)$ corresponds to position along the colon from cecum to rectum and the vertical axis $(y)$ corresponds to rotation around the circumference of the colon. Each pixel of $I_{i}$ has an interpolated value of the corresponding shape index $S I$ from the three-dimensional surface $S_{i}$. SI is defined as

$$
S I \equiv \frac{1}{2}-\frac{1}{\pi} \arctan \left(\frac{\kappa_{1}+\kappa_{2}}{\kappa_{1}-\kappa_{2}}\right),
$$

where $\kappa_{1}$ and $\kappa_{2}$ are the principal curvatures extracted from $S_{i}$. Furthermore, any other measure of local curvature or other voxel grey value statistics derived from the original CT scan could be associated with a pixel in $I_{i}$. The interpolated value is always computed based on the three corner values of each triangle in $D_{i}$ which correspond to the $3 \mathrm{D}$ vertices of $S_{i}$. The resulting supine and prone raster-images with a resolution of $n_{x}=3000$ and $n_{y}=150$ are shown in Fig. 3 (top) and Fig. 3 (middle).

\subsection{Establishing Spatial Correspondence between Prone and Supine}

The 2D manifolds are used to generate shape index images which are first aligned in the $y$-direction to account for differences in the $0^{\circ}$ position arbitrarily assigned by the planar embedding. This is performed automatically by applying a circular shift in $y$-direction to $I_{1}$ that minimises the Sum of Squared Differences (SSD) between $I_{1}$ and $I_{2}$. The B-spline registration is currently performed on the flat Euclidean plane and not in a cylindrical framework. Therefore $I_{1}$ and $I_{2}$ are 


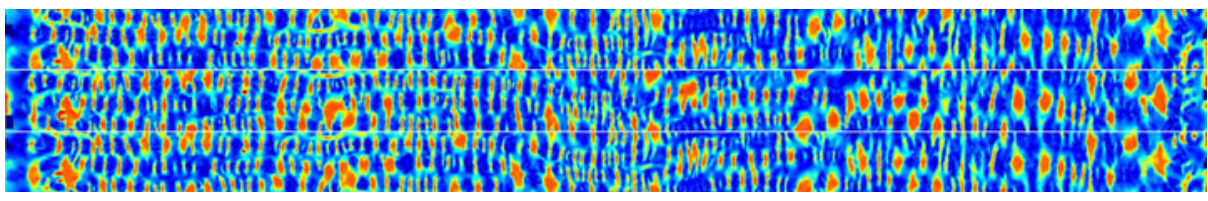

Fig. 3. Supine (top), prone (middle) and deformed supine (bottom) repeated rasterimages where each pixel has the value of the corresponding shape index from the inner colonic surface

repeated in the $y$-direction, resulting in images with a resolution of $N_{x} \times N_{y}=$ $n_{x} \times 2 \cdot n_{y}$, in order to simulate the cylindrical images during the registration.

A $2 \mathrm{D}$ B-spline registration is then performed with the shifted $I_{1}$ as target and $I_{2}$ as source. We use the fast implementation provided by 13 . The registration is performed in two stages, the first to recover the larger deformations, and the second to recover the finer deformations. The first stage consists of five resolution levels. The second stage consists of three resolution levels and uses the result from the first stage as the starting transformation for the coarsest level. Both the image and B-spline control point grid resolutions are doubled at each level. The final resolution level uses images with $3000 \times 300\left(n_{x} \times 2 \cdot n_{y}\right)$ pixels and control points spaced every 12.5 pixels in both directions. SSD is used as the similarity measure. The gradient of the cost function is smoothed at each iteration using a Gaussian kernel with a standard deviation of three control points for the first stage and one control point for the second. No additional penalty term is used for the first stage but bending energy is used for the second. Gaussian smoothing of the $2 \mathrm{D}$ images is applied at each resolution level during the first stage of registration but is not used for the second.

The central half of the B-spline registration result, from $25 \%$ to $75 \%$ of the $y$-axis, covers the whole inner colon surface $\left(0^{\circ}\right.$ to $\left.360^{\circ}\right)$ and should have a similar displacement at $y=0.25 \cdot N_{y}$ and $y=0.75 \cdot N_{y}$ thanks to the duplication of image data in the $y$-direction prior to registration. To force the result to be fully cylindrical, such that the transformation is continuous from $y=0.25 \cdot N_{y}$ to $y=0.75 \cdot N_{y}$, the control points displacements at $y=0.25 \cdot N_{y}$ and $y=0.75 \cdot N_{y}$ are averaged together, and the control point displacements before $y=0.25 \cdot N_{y}$ and after $y=0.75 \cdot N_{y}$ are replaced with the corresponding control point displacements from the central section. This results in a continuous transformation around the entire inner colon surface and allows the mapping between $S_{1}$ and $S_{2}$. From this mapping it is straightforward to determine the full 3D mapping $f$, as shown in Fig. 1.

\section{Clinical Evaluation}

Ethical permission and informed consent to utilise anonymised CT colonography datasets was obtained. Colonic cleansing and insufflations were undertaken for all 
twelve subjects used in this study in accordance with current recommendations 14. We selected patients where the colon was fully distended in the prone and supine scan with either fluid tagging or little remaining fluid in order to provide complete segmentations. The radiologist (with experience in over 500 validated colonography studies) matched pairs of reference points from the original prone and supine CT slices. Using separate multiplanar reformats, a combination of polyps, normal anatomical structures and diverticula were identified from multiple colonic segments, resulting in approximately ten pairs of coordinates per patient.

\subsection{Human Observer Error Estimation}

The error of establishing the correspondence points in both images was determined by repeating the validation following an interval of seven days for patients 1 to 6 (in order to reduce recall bias). The repeated validation was performed using the original coordinates from the supine datasets, and the radiologist was asked to re-identify the corresponding points in the prone dataset. The results suggest a significant difficulty in finding correct correspondences in the prone and supine CT-images, which provides further justification for a method of establishing prone-supine correspondence. We removed outliers based on the maximum likelihood estimate [15, p. 202] using the median $\sigma=1.4828(1+5 /(n-3))$ med $|E|$, where the inliers are defined by $E_{i} \leq \gamma$ and $\gamma=1.96 \sigma$. This gives a threshold $\gamma$ of $14.6 \mathrm{~mm}$ in order to get reliable landmarks to validate the registration and reduces the human observer error from $(8.2 \pm 12.5) \mathrm{mm}$ to $(3.8 \pm 2.9) \mathrm{mm}$. The remaining prone landmarks are averaged and used to validate the registration for the first six patients.

\subsection{Registration Error}

In order to determine the registration error we find the closest surface points on $S_{1}$ to the landmarks in the prone image. We then find the closest points on $S_{2}$ to the corresponding landmarks in the supine image, transform them using the $3 \mathrm{D}$ mapping $f$, and calculate the distance to the points on $S_{1}$.

In order to access the accuracy of the method, we had to remove 11 reference points which were outliers (determined for the first six patients) and 3 points which clearly failed to align correctly. Just using the direct mapping between $D_{1}$ and $D_{2}$ after initial alignment in the $y$-direction resulted in an error for the remaining 120 data points of $(23 \pm 15) \mathrm{mm}$. After establishing the spatial correspondence using the B-spline registration, the error was reduced to $(9.0 \pm 11) \mathrm{mm}$. The histogram of the registration error shows that the majority (91 \%) lie below $20 \mathrm{~mm}$. Successful registration could be achieved for 8 out of 12 polyps (distributed over 8 patients) with an accuracy of $(4.7 \pm 4.1) \mathrm{mm}$. These are promising results and have potential clinical impact to the screening process.

Four patients have clearly higher errors which correspond to the data points larger than $20 \mathrm{~mm}$ in Fig. 4. It appears that some of these errors may be due to some of the corresponding landmarks being assigned wrongly, e.g.to a neighboring fold. The excluded reference points were one haustral fold and the two 


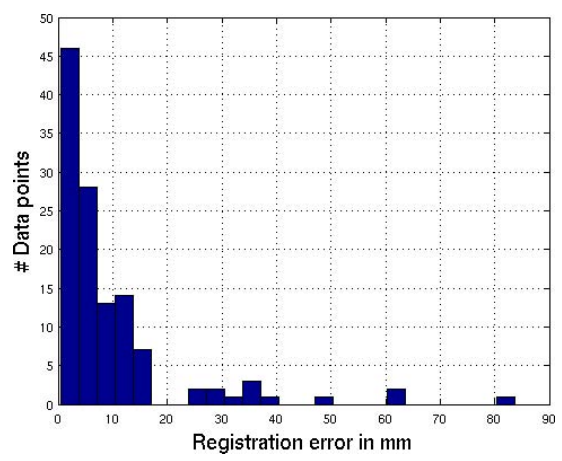

Fig. 4. Histogram of the registration error in $\mathrm{mm}$

unsuccessfully registered polyps which lay in the rectum or cecum and had a large error due to the deformations in the parameterisation in that region.

\section{Summary and Future Work}

We propose a novel registration method for establishing spatial correspondence for the inner colon surface extracted from prone and supine CT colon images. Our registration process works by using an appropriate indexing system which reduces the registration task from a $3 \mathrm{D}$ - to a $2 \mathrm{D}$-problem. This is done with a one-to-one conformal mapping of the entire inner colon surfaces to a parameterisation where one dimension corresponds to distance along the colon and the other to its angular orientation. From that parameterisation we compute images which correspond to 3D positions and their shape indices computed on $3 \mathrm{D}$ surfaces. This allows a non-rigid registration of the prone and supine colon surfaces which can handle the large deformations occurring between both positions. Furthermore, the provided framework could easily be extended to include any statistic or set of statistics derived from the original CT-images.

Future work will include phantom studies and other synthetic data for a more reliable gold standard to be used in the validation of the method. The $2 \mathrm{D}$ B-spline registration will be implemented in a truly cylindrical way such that repeating the images will not be necessary. We will experiment with additional statistics derived from the original CT volumes with the aim of increasing the accuracy of the method. Furthermore, we will investigate automated topological correction of the colon segmentation. This is in order to increase robustness and deal with insufficiently distended colons where the colon surface could be collapsed.

It is clear that a major limitation of this study is the lack of a sufficiently accurate gold standard. The reported accuracy of our results is almost certainly limited by observer error in picking corresponding landmarks in prone and supine CT. To address this we are building a physical phantom from porcine bowel with embedded CT visible markers to provide an accurate gold standard. 


\section{References}

1. WHO: Cancer (2006), http://www .who.int/mediacentre/factsheets/fs297/en/ (retrieved on July 2010)

2. Rex, D., Cutler, C., Lemmel, G., Rahmani, E., Clark, D., Helper, D., Lehman, G., Mark, D.: Colonoscopic miss rates of adenomas determined by back-to-back colonoscopies. Gastroenterology 112(1), 24-28 (1997)

3. Acar, B., Napel, S., Paik, D., Li, P., Yee, J., Beaulieu, C., Jeffrey, R.: Registration of supine and prone ct colonography data: Method and evaluation. Radiology 221, 332 (2001)

4. Näppi, J., Okamura, A., Frimmel, H., Dachman, A., Yoshida, H.: Region-based supine-prone correspondence for reduction of false positive cad poly candidates in ct colonography. Acadamic Radiology 12, 695-707 (2005)

5. Suh, J., Wyatt, C.: Deformable Registration of Supine and Prone Colons for Computed Tomographic Colonography. Journal of computer assisted tomography 33(6), $902(2009)$

6. Lamy, J., Summers, R.: Intra-patient colon surface registration based on teniæ coli. In: SPIE Medical Imaging, pp. 116-123 (2007)

7. Johnson K., Johnson C., F.J.M.R.S.R.: CT colonography using 360-degree virtual dissection: a feasibility study. AJR Am J Roentgenol. 186 (2006) 90-95

8. Jin, M., Kim, J., Luo, F., Gu, X.: Discrete surface ricci flow. IEEE Transactions on Visualization and Computer Graphics 14(5), 1030-1043 (2008)

9. Qiu, F., Fan, Z., Yin, X., Kaufman, A., Gu, X.: Colon Flattening with Discrete Ricci Flow. In: MICCAI workshop, pp. 97-102 (2008)

10. Hamilton, R.: Three-manifolds with positive Ricci curvature. J. Differential Geom. 17(2), 255-306 (1982)

11. Chow, B., Luo, F.: Combinatorial Ricci flows on surfaces. J. Differential Geom. 63(1), 97-129 (2003)

12. Slabaugh, G., Yang, X., Ye, X., Boyes, R., Beddoe, G.: A Robust and Fast System for CTC Computer-Aided Detection of Colorectal Lesions. Algorithms 3(1), 21-43 (2010)

13. Modat, M., Ridgway, G., Taylor, Z., Lehmann, M., Barnes, J., Hawkes, D., Fox, N., Ourselin, S.: Fast free-form deformation using graphics processing units. Computer Methods and Programs in Biomedicine (2009)

14. Taylor, S., Laghi, A., Lefere, P., Halligan, S., Stoker, J.: European Society of Gastrointestinal and Abdominal Radiology (ESGAR): consensus statement on CT colonography. European Radiology 17(2), 575-579 (2007)

15. Rousseeuw, P., Leroy, A.: Robust regression and outlier detection. Wiley-IEEE (2003) 